\title{
Multivariate Statistical Analysis Tool for the Interpretation and the Quantification of Hyperspectral Data: Application to 3D EDX/FIB Images
}

\author{
G. Lucas, P. Burdet, M. Cantoni, and C. Hébert \\ Ecole Polytechnique Fédérale de Lausanne (EPFL), Interdisciplinary Centre for Electron \\ Microscopy (CIME), CH 1015 Lausanne, Switzerland.
}

Over the past few years, capabilities of microanalytical instruments have largely improved, leading to a huge amount of raw data especially for 3D analysis, which are usually difficult to interpret without post-processing. Multivariate Statistical Analysis (MSA), and in particular factor analysis allows to reduce the dimensionality of the data and to simplify their interpretation [1]. Moreover it can greatly improve the accuracy of the quantification by removing the noise components from the data and provide a significant speedup. A software tool has been developed to take advantage of the MSA in microanalysis.

To illustrate its usage, a sample produced by laser welding of a nickel-titanium alloy wire and a stainless steel wire [5] has been analyzed at the crack nucleation site by scanning electron microscopy and energy-dispersive X-ray spectroscopy (EDX) coupled with a focused ion beam (FIB) to investigate the material slice by slice. In order to assess in the SEM the method a prior 2D map has been performed with one hour of acquisition time, insuring a large number of detector counts, i.e. a good statistic. However in order to keep a reasonable acquisition time, each map of the 3D dataset have been carried out in six minutes. The final 3D spectral data are consequently subject to an important noise.

In a first step weighted principal component analysis (PCA) based on singular value decomposition (SVD) has been applied on the EDX data, providing abstract components with no physical meaning [2]. By selecting the components responsible for the largest part of the variance in the data, the dataset can be reconstructed without the noise components. Then factor rotations in spatial and spectral domains of the noise-free components have been performed [3]. The goal of the rotations is to maximize the variance of the spectral or the spatial factors, in other words to enhance the contrast of one domain at the expense of the other. In our case, the resulting factors can be almost directly interpreted in terms of elements and compounds, respectively for spectral-domain and spatialdomain rotations. Figure 1 shows some of the spectral and spatial factors after rotation in spectraldomain in the $2 \mathrm{D}$ case. In a last step selected rotated factors corresponding to $\mathrm{Fe}, \mathrm{Ni}$ and $\mathrm{Ti}$ elements have been used to isolate the different phases present in the microstructure, by relating the spatial components in a ternary histogram, as shown on figure 2. By defining groups of pixels having similar composition, the data can then be easily segmented.

The segmentation reveals a succession of phases corresponding to a diffusion path occurring during the welding process. Results are in excellent agreement with the EDX quantification using standards carried out on the same data set [4], demonstrating the strength of rotated SVD-PCA for spectral data analysis. It is also remarkable that the quantification itself can be significantly improved using PCA, not only because of the noise reduction, but also because quantification algorithms can be applied efficiently on a reduced dataset, hence decreasing significantly the computation time. 


\section{References:}

[1] E. R. Malinowski, Factor Analysis in Chemistry ( $3^{\text {rd }}$ Ed.), Wiley, New York, 2002.

[2] N. Bonnet et al., Ultramicroscopy, 77 (1999) 97.

[3] M. R. Keenan et al., Surf. Interface Anal., 41 (2009) 79.

[4] P. Burdet et al., to be published.

[5] Acknowledgments: sample has been kindly provided by J. Vannod (LSMX, EPFL).

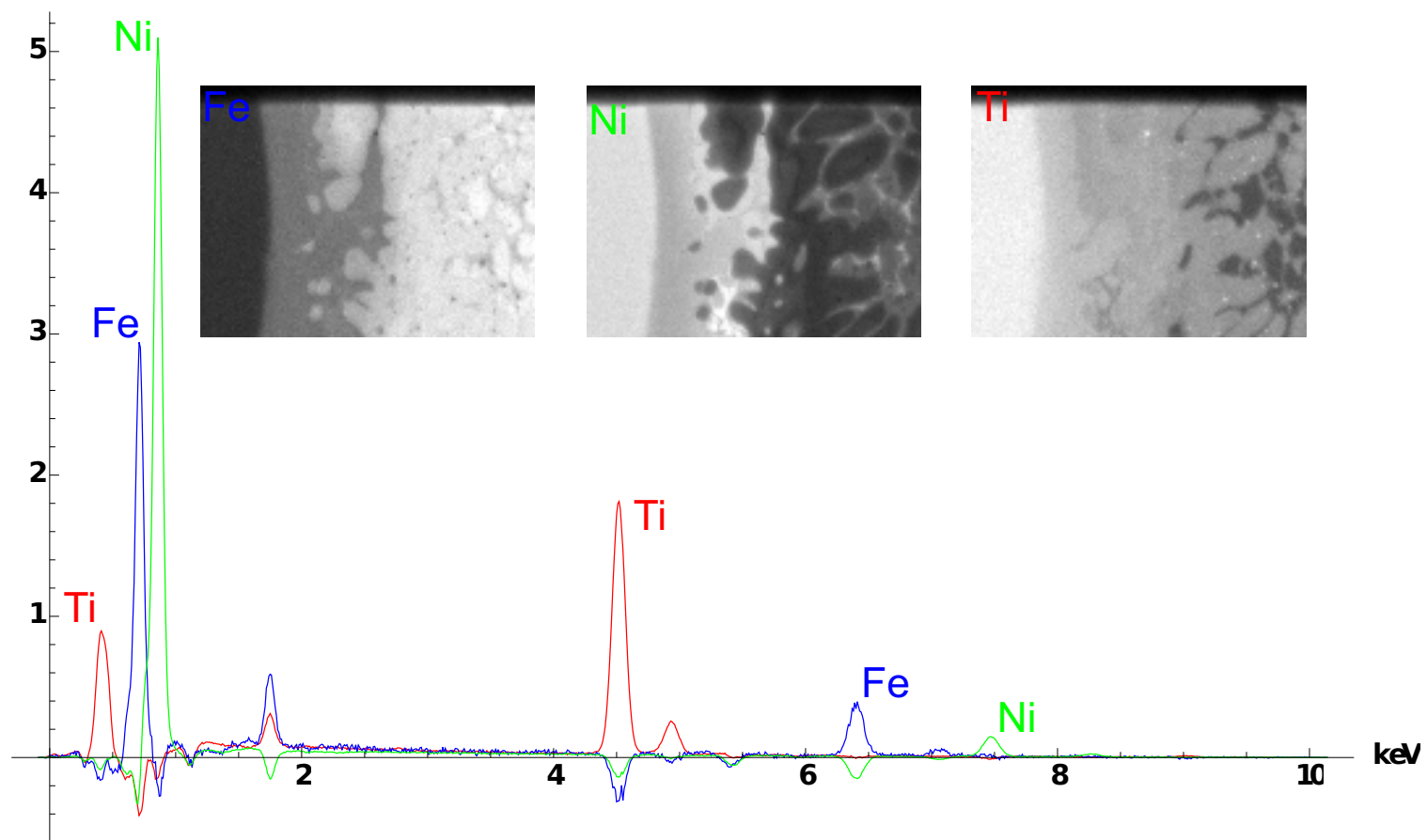

Fig. 1. Spectral and spatial components after rotation in spectral domain, corresponding to $\mathrm{Fe}, \mathrm{Ni}$ and $\mathrm{Ti}$.

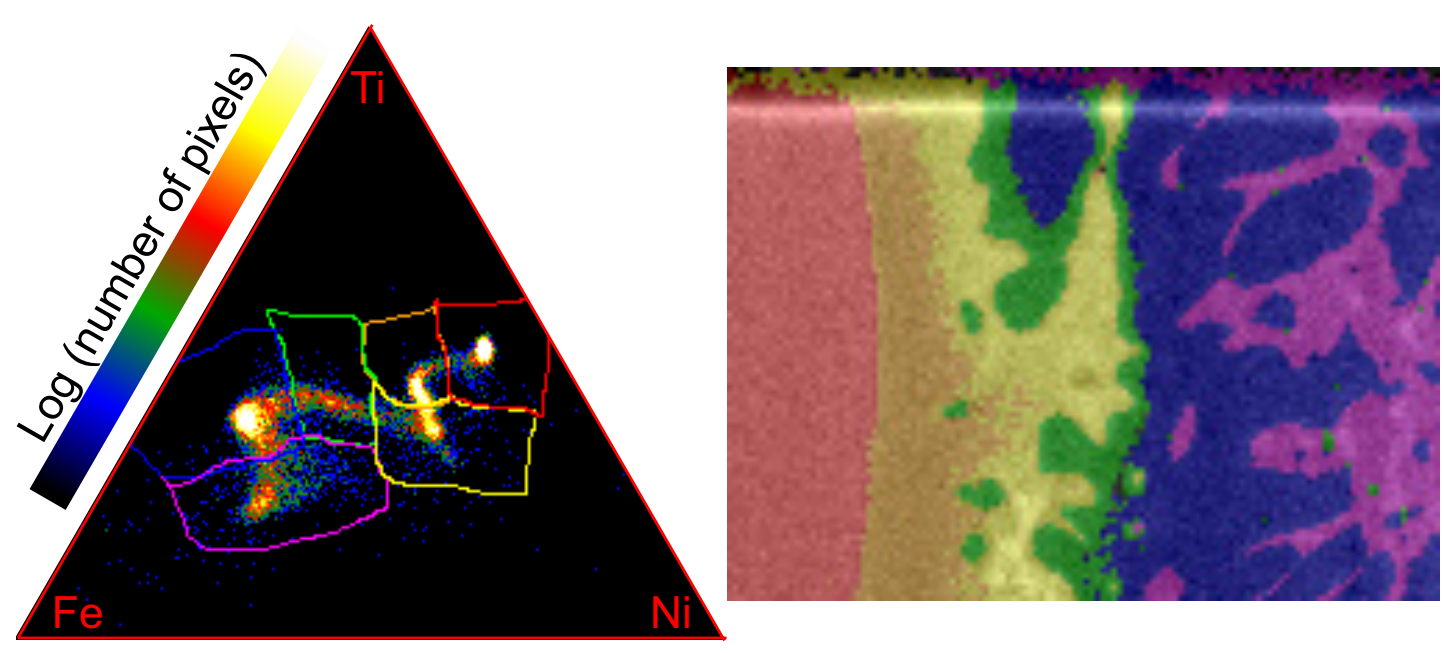

Fig. 2. On the left side, the ternary histogram relating spatial components having $\mathrm{Fe}, \mathrm{Ni}$ and $\mathrm{Ti}$ characters. On the right side, the phase segmentation obtained using selected areas on the histogram. 